\title{
High-Resolution Cathodoluminescence Investigation of Degradation Processes in InGaN Green Laser Diodes
}

David Gachet $^{1}$, Carlo de Santi ${ }^{2}$, Matteo Meneghini ${ }^{2}$, Giovanna Mura ${ }^{3}$, Massimo Vanzi ${ }^{3}$, Gaudenzio Meneghesso $^{2}$ and Enrico Zanoni ${ }^{2}$

1. Attolight AG, EPFL Innovation Park, Building D, CH-1015 Lausanne, Switzerland.

2. Department of Information Engineering, University of Padova, Padova, Italy.

3. Department of Electrical and Electronic Engineering, University of Cagliari, Cagliari, Italy.

III-nitride materials have revolutionized optoelectronics as they are behind UV-blue LEDs and laser diodes [1]. In most III-nitride optoelectronic devices, light emission arises from InGaN multi quantum wells (MQW) layers. Increasing the In fraction in InGaN MQWs allows reaching green emission (500$550 \mathrm{~nm}$ ) and thus closing the 'green gap', with potential applications in biomedical science and miniaturized high brightness beamers. However, increased incorporation of In leads to limited reliability of green LEDs and lasers even under moderate operating conditions [2].

Here we aim at investigating the physical processes responsible for the degradation of commercial InGaN based green laser diodes. The laser diodes under study have been stressed under a controlled way and their threshold current has been systematically monitored at different stages of the stress process. Such measurements give a first insight into the degradation process at work in the green laser diodes. However, they do not give much information about the microscopic processes involved here.

To overcome this limitation, we have applied high spatial and spectral resolution cathodoluminescence (CL) spectroscopy in a scanning electron microscope (SEM). SEM-CL offers several advantages over usual optical spectroscopy techniques including nanoscale spatial resolution combined with surface morphology information [3]. Top view and cross section view (at the level of the output facet - Fig. 1b and $\mathrm{c}$ - or after focused ion beam preparation - Fig. 2a) studies were conducted at different stages in the degradation process. Hyperspectral SEM-CL imaging allowed capturing the local behaviour of all layers making part of the laser diodes (Fig. 2), and this at different stages in the degradation process.

These extensive SEM-CL studies have shown that the MQWs are not the only layers to be degraded (as initially thought). InGaN waveguide as well as $\mathrm{n}-\mathrm{AlGaN}$ cladding layer are also subject to degradation. Degradation has been revealed by a combined local decrease in the intensity of the CL signal and fluctuation in the emission wavelength in the aforementioned layers. The degradation process occurs under the ridge of the laser diodes where the injected current reaches its maximum value and the optical confinement takes place.

SEM-CL has proved here to be a powerful tool to unravel the microscopic origin of degradation processes in semiconductor laser structures. In fact, CL microscopy is a technique of choice for failure analysis of optoelectronic devices and electronic components [4].

References:

[1] Nakamura et al, Jpn J. Appl. Phys. 34 (1995) p. L797.

[2] Marioli et al, Physica Status Solidi A 212 (2015), p. 974. 
[3] L. Reimer in "Scanning Electron Microscopy", (Springer, Berlin) Ch. 1.

[4] The authors acknowledge Michael Marioli for performing part of the stress tests.
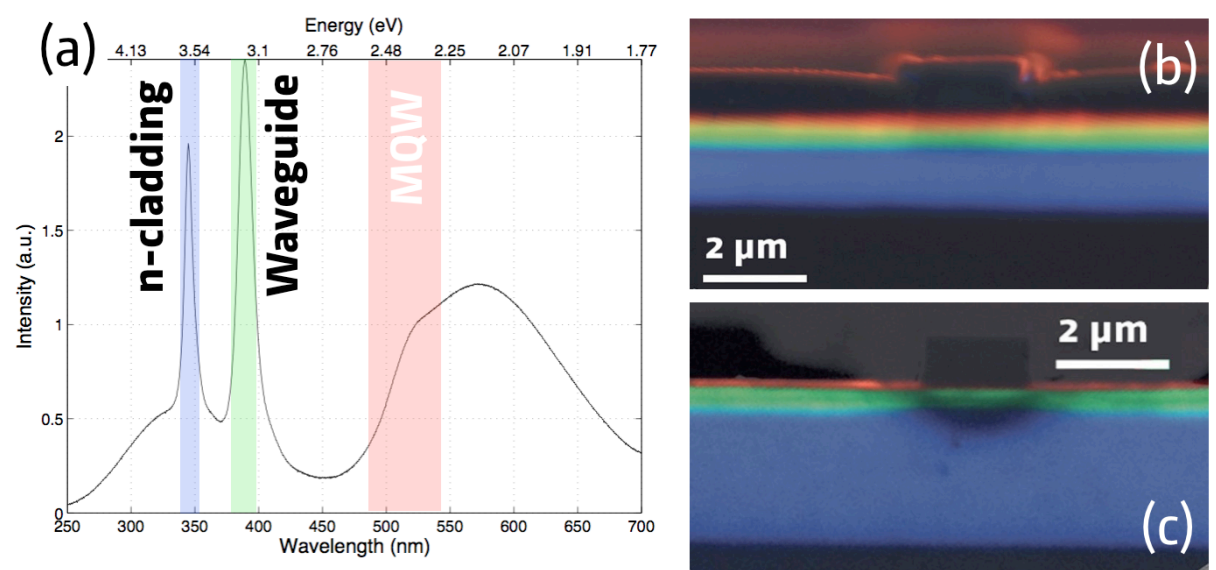

Figure 1. (a) Averaged-CL spectrum of an unstressed laser diode at the level of the output facet. (b,c) Monochromatic CL images of the output facet of an unstressed (b) and a degraded laser diode (c). The color coding (RGB) in (b) and (c) corresponds to the spectral bands depicted in (a).

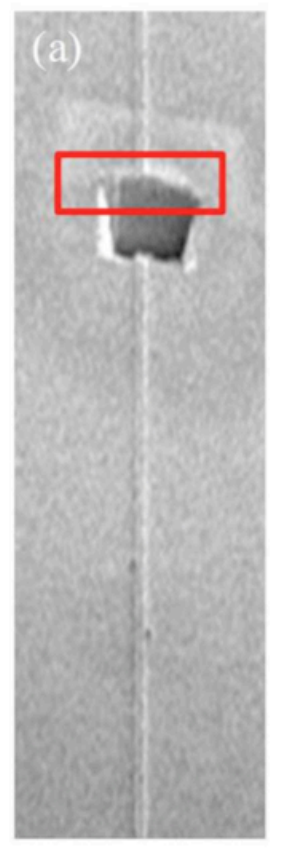

(b)

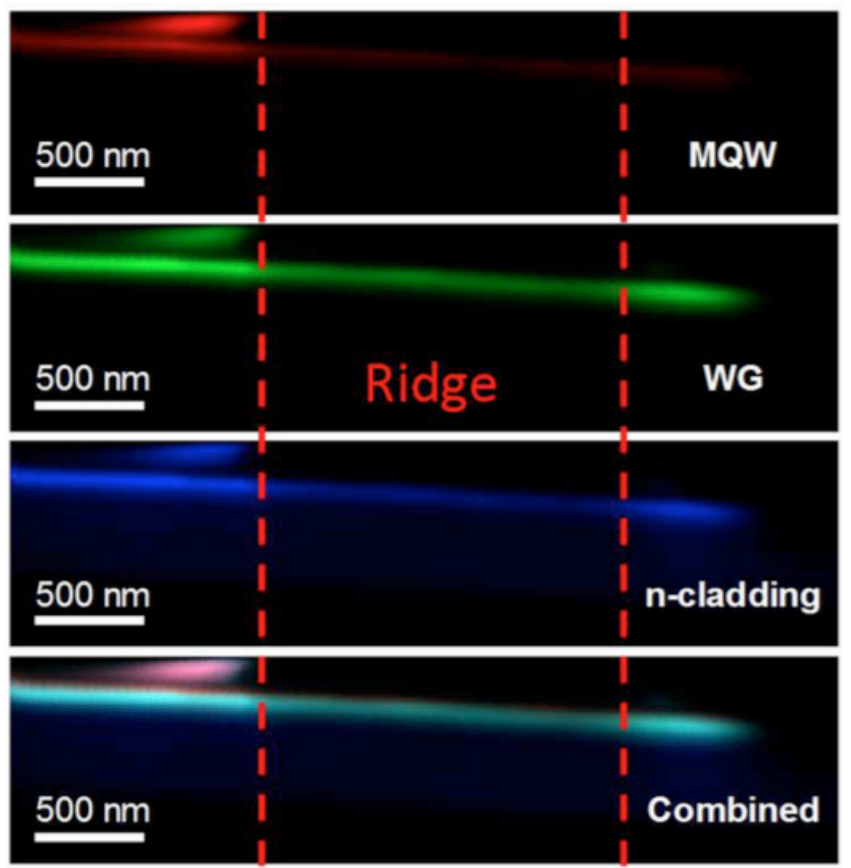

Figure 2. (a) Secondary electron image of the area where focused ion beam preparation (FIB) has been performed in a degraded laser. (b) Monochromatic CL images of the various layers under the laser ridge. MQW: InGaN multi-quantum wells; WG: InGaN waveguide. 